\title{
Fabrication of Poly(p-oxybenzoyl) Nanowhisker
}

\author{
Kunio KimURA, ${ }^{\dagger}$ Shinichi YamaZAKI, Yoshiaki MatsuOKA, and Yuhiko Yamashita \\ Faculty of Environmental Science and Technology, Okayama University, \\ 3-1-1 Tsushima-naka, Okayama 700-8530, Japan
}

(Received June 21, 2005; Accepted August 15, 2005; Published December 15, 2005)

\begin{abstract}
Fabrication of poly(p-oxybenzoyl) (POB) nanowhiskers was examined by using reaction-induced crystallization of oligomers during the polymerization of $p$-acetoxybenzoic acid in liquid paraffin. Width of the POB whiskers was susceptible to polymerization temperature and it was efficiently controlled by a change in temperature during the polymerization. Increase in polymerization temperature just after nucleation (Han) reduced the degree of super saturation of oligomers $(\sigma)$ resulting in the depression of the width increase by the crystallization of oligomers on side surface of the whiskers. Temperature drop just before nucleation (Cbn) enhanced the value of $\sigma$ resulting in the generation of much more nuclei having the smaller width. On the basis of these results, the combination of these two methods Han and Cbn afforded the whiskers having the smallest width, and the POB nanowhiskers were prepared of which the width and the length were $190 \mathrm{~nm}$ and $18.6 \mu \mathrm{m}$, respectively. [DOI 10.1295/polymj.37.906]

KEY WORDS Poly( $p$-oxybenzoyl) / Nanowhisker / Polyester / Crystallization / Oligomer /
\end{abstract}

One-dimensional nanoscale materials such as nanowhiskers, nanofibers, nanotubes and nanobelts are attracting attention because of their intriguing chemical and physical properties, and actively studied to create novel devices. ${ }^{1-3}$ Among them, nanowhiskers of inorganic compounds are recently developed for electronic/optoelectronic devices and sensors. ${ }^{4,5}$ Polymer nanowhiskers and nanofibers have also been received much attention as very interesting one-dimensional materials. ${ }^{6-8}$ The polymer nanofibers are usually fabricated by the polymer nano-processing technologies represented by electrospinning ${ }^{9-15}$ or the self-assembling of polymer molecules. ${ }^{16-18}$ Template syntheses have been developed involving the introduction of the materials within channels of templates. ${ }^{19-25}$ In contrast to this, the fabrication of polymer nanowhiskers is more difficult, and to our knowledge $\mathrm{C}_{60}$ whisker is the only nanowhisker prepared by a liquid-liquid interfacial precipitation under light illumination. ${ }^{26}$

Rigid-rod aromatic polymers are characterized by the unique combination of thermal and mechanical properties, which makes them useful candidates for high performance materials. ${ }^{27}$ Although the nanowhiskers and nanofibers of rigid aromatic polymers have a bright prospect of valuable industrial materials, they show usually neither solubility nor feasibility and hence it is difficult to process them by the previous fabrication procedures. The fabrication method of them has not been reported so far.

Poly(p-oxybenzoyl) (POB) which is a wholly aromatic polyester possesses the aforesaid antagonistic problem between properties and processability. In order to overcome this problem, we have been studying the morphology control of POB by using crystallization of oligomers during polymerization, and succeeded in obtaining POB whiskers by the polymerization of $p$-acetoxybenzoic acid (ABA) in liquid paraffin (LPF) with the elimination of acetic acid without stirring. ${ }^{28}$ The $\mathrm{POB}$ whisker was a microscale single crystal in which the extended polymer chains were aligned along the long axis of the whiskers. This preparation technique stipulated a useful method for morphology control of intractable polymers and many other polymer whiskers have been prepared. ${ }^{29-37}$ Length control of the whiskers was of great importance in industrial application and the method to lengthen the POB whiskers was developed by the continuous addition of oligomers. ${ }^{38}$ If width of the POB whiskers can be controlled and shortened to nanoscale, it will provide a new methodology for fabrication of nanowhiskers of intractable rigid polymers.

This article examines the influence of the polymerization temperature on the size of the POB whiskers, in particular the width, and describes the new finding on fabrication of the POB nanowhiskers.

\section{EXPERIMENTAL}

\section{Materials}

ABA was purchased from Tokyo Kasei Co. Ltd. and purified by recrystallization from ethyl acetate. LPF was purchased from Nacalai Tesque Co. Ltd. and purified by vacuum distillation $\left(220-240^{\circ} \mathrm{C} / 0.2 \mathrm{mmHg}\right)$.

\section{Polymerization Method}

The polymerization methods in which the tempera-

${ }^{\dagger}$ To whom correspondence should be addressed (Tel/Fax: +81-086-251-8902, E-mail: polykim@cc.okayama-u.ac.jp). 




Figure 1. Polymerization temperature profiles.

ture was dropped down to lower temperature just before and after the solution became turbid due to the nucleation were named as "Cooling before nucleation (Cbn)" and "Cooling after nucleation (Can)," respectively. On the other hand, the polymerization method in which the temperature was jumped up to higher temperature just after the solution became turbid was named as "Heating after nucleation (Han)." The heating rate was $5^{\circ} \mathrm{C} \cdot \mathrm{min}^{-1}$ and the cooling rate was $2{ }^{\circ} \mathrm{C} \cdot \mathrm{min}^{-1}$. The polymerization method in which the polymerization temperature was constantly kept was abbreviated as "Const." The temperature profiles of these methods are presented in Figure 1. $\Delta T$ describes the value of temperature change as shown in Figure 1. The monomer concentration was $1.5 \%$. The polymerization procedure by the combination of Cbn and Han was typically described as follows.
Preparation of Whiskers by Cbn and Han $(T=320$ $\left.{ }^{\circ} \mathrm{C}, \Delta T_{b n}=-60{ }^{\circ} \mathrm{C}, \Delta T_{a n}=+90^{\circ} \mathrm{C}\right) . \quad \mathrm{ABA}(0.9 \mathrm{~g}$, $5.0 \mathrm{mmol}$ ) and $60 \mathrm{~mL}$ of LPF were placed into a cylindrical polymerization reactor equipped with a thermometer, a mechanical stirrer, and gas inlet and outlet tubes. This reaction mixture was heated with stirring. When ABA was entirely dissolved, the stirring was stopped. Then the mixture was heated up to $320^{\circ} \mathrm{C}$ and maintained at this temperature under a slow stream of nitrogen. After one minute, the reaction solution was allowed to cool at $260^{\circ} \mathrm{C}$ with the rate of $2{ }^{\circ} \mathrm{C} \cdot \mathrm{min}^{-1}$. The solution became turbid after $5 \mathrm{~min}$ at $260^{\circ} \mathrm{C}$ due to the nucleation and then the reaction temperature was raised to $350{ }^{\circ} \mathrm{C}$ with the rate of 5 ${ }^{\circ} \mathrm{C} \cdot \mathrm{min}^{-1}$. The reaction was continued at $350{ }^{\circ} \mathrm{C}$ for $6 \mathrm{~h}$. The whiskers were collected by filtration at $350^{\circ} \mathrm{C}$ in the nitrogen atmosphere, washed several times with $n$-hexane and acetone, and then dried in a vacuum oven at $50^{\circ} \mathrm{C}$ for overnight. The whiskers were obtained with the yield of $42 \%$. FT-IR $(\mathrm{KBr})\left(\mathrm{cm}^{-1}\right)$ : 3074, 2923, 1739, 1597, 1508, 1415, 1255, 1199, 1155, 1049, 1011, 885, 758, 673. Anal. Calcd for $\mathrm{C}_{7} \mathrm{H}_{4} \mathrm{O}_{2}$ : C, 70.00; H, 3.36. Found: C, 70.21; H, 3.19.

\section{Measurements}

Morphological observation was performed by a Hitachi S-2150 scanning electron microscopy. Samples for the observation were dried, sputtered with gold and observed at $20 \mathrm{kV}$. Average shape parameters of the whiskers were determined by taking the average of over 80 observation values. The POB whiskers exhibited hexagonal cross section. From the shape parameters obtained, the number of the whiskers $(N)$ was estimated by using the following equation.

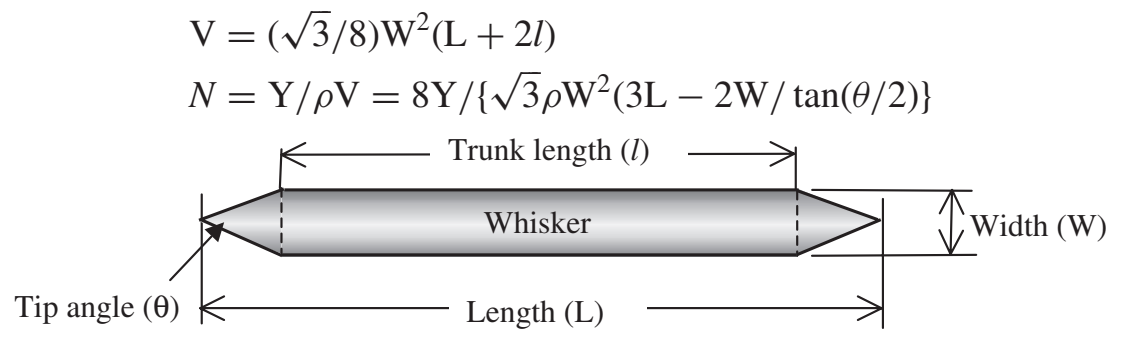

where $\mathrm{V}$ : the volume of the whisker, W: width of whiskers, L: length of whiskers, $l$ : trunk length of whiskers, $\theta$ : tip angle of whiskers, Y: yield of whiskers, $\rho$ : density of whiskers.

$\rho$ of the whiskers was $1.52 \mathrm{~g} \mathrm{~cm}^{-3}$ measured by the floatation method using bromoform and toluene at $25^{\circ} \mathrm{C}$.

\section{RESULTS AND DISCUSSION}

\section{Concept for Width Control of POB Whiskers}

The width control in the present study is conceptu- ally simple. As previously reported, ${ }^{28,39}$ the oligomers are formed in the solution by the condensation reaction of ABA with eliminating acetic acids. LPF is a poor solvent to $\mathrm{POB}$, and therefore the oligomers are precipitated through the super-saturated state when the degree of polymerization exceeds critical value of 8 . The resulting oligomers are crystallized in the form of lamellae and the lamellae stack up to 

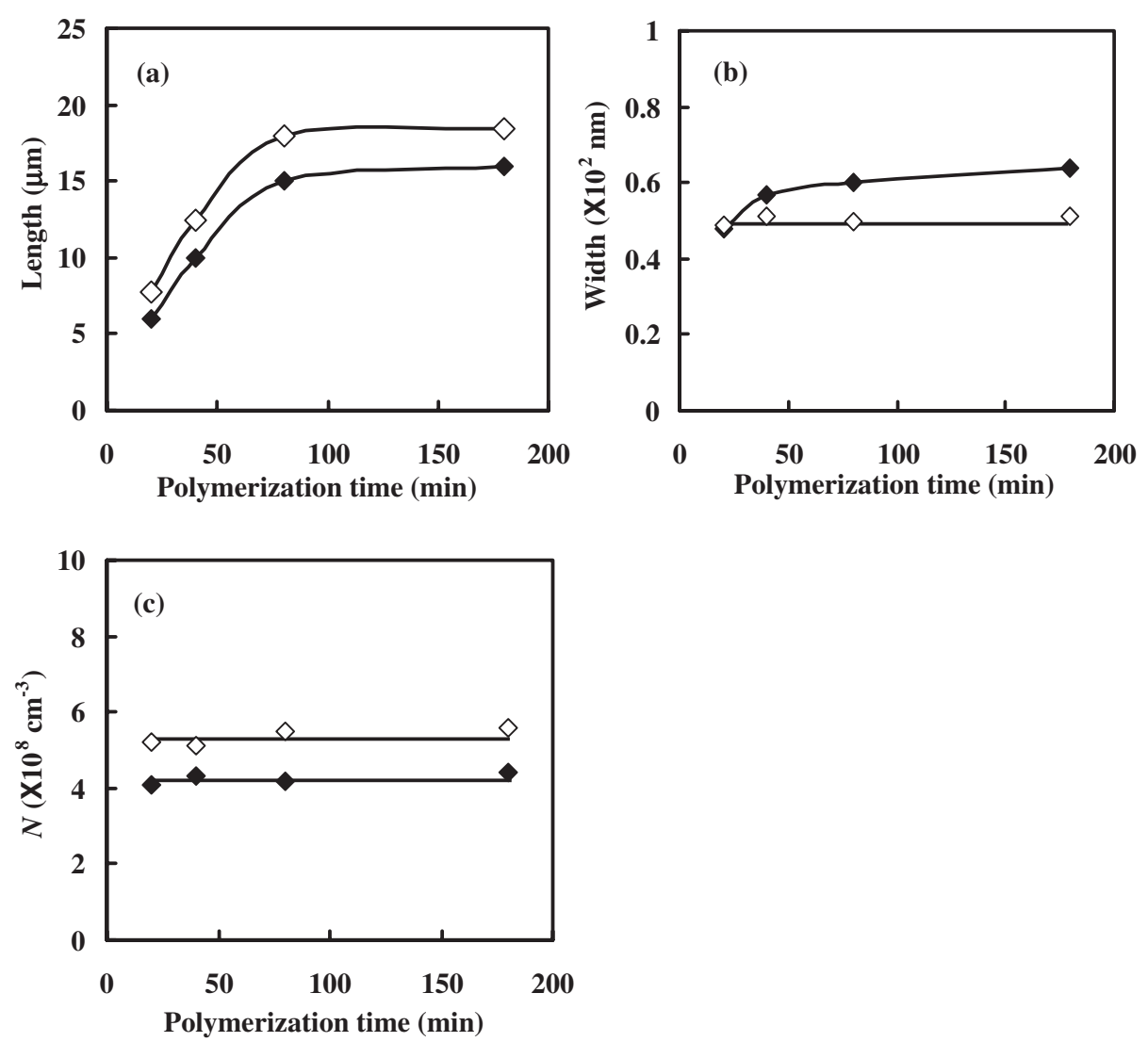

Figure 2. Plots of (a) average length, (b) width and (c) number of POB whiskers $(N)$ prepared in Run No. $1(\diamond)$ and $2(\diamond)$ as a function of time.

Table I. Results of polymerization

\begin{tabular}{|c|c|c|c|c|c|c|c|c|c|}
\hline \multirow{2}{*}{$\begin{array}{l}\text { Run } \\
\text { No. }\end{array}$} & \multicolumn{4}{|c|}{ Polymerization conditions } & \multirow{2}{*}{$\begin{array}{c}\text { Polymer } \\
\text { yield } \\
(\%)\end{array}$} & \multicolumn{3}{|c|}{ Whisker size ${ }^{d}$} & \multirow{2}{*}{$\begin{array}{c}N^{\mathrm{e}} \\
\left(10^{8} \mathrm{~mL}^{-1}\right)\end{array}$} \\
\hline & Method $^{\mathrm{a}}$ & $\begin{array}{l}T_{\mathrm{i}}^{\mathrm{b}} \\
\left({ }^{\circ} \mathrm{C}\right)\end{array}$ & $\begin{array}{l}\Delta T_{\mathrm{bb}}{ }^{\mathrm{c}} \\
\left({ }^{\circ} \mathrm{C}\right)\end{array}$ & $\begin{array}{l}\Delta T_{\text {an }}{ }^{\mathrm{c}} \\
\left({ }^{\circ} \mathrm{C}\right)\end{array}$ & & $\begin{array}{l}\text { Length } \\
(\mu \mathrm{m})\end{array}$ & $\begin{array}{l}\text { Width } \\
\text { (nm) }\end{array}$ & $\begin{array}{l}\text { Tip angle } \\
\text { (degree) }\end{array}$ & \\
\hline 1 & Const & 320 & 0 & 0 & 40 & $17.6(24 \%)$ & $730(25 \%)$ & 12 & 4.4 \\
\hline 2 & Han & 320 & 0 & +30 & 33 & $21.4(22 \%)$ & $500(33 \%)$ & 8 & 5.6 \\
\hline 3 & Can & 320 & 0 & -30 & 24 & $13.2(38 \%)$ & $1150(51 \%)$ & $-^{\mathrm{f}}$ & $-^{f}$ \\
\hline 4 & Cbn & 320 & -60 & 0 & 31 & $8.8(21 \%)$ & $270(45 \%)$ & 9 & 50 \\
\hline 5 & Const & 260 & 0 & 0 & 18 & $5.9(22 \%)$ & $440(32 \%)$ & $-^{f}$ & $-^{f}$ \\
\hline 6 & Cbn \& Han & 320 & -60 & +60 & 36 & $16.0(23 \%)$ & $220(40 \%)$ & 7 & 46 \\
\hline 7 & Cbn \& Han & 320 & -60 & +90 & 42 & $18.6(26 \%)$ & $190(36 \%)$ & 6 & 55 \\
\hline
\end{tabular}

${ }^{\mathrm{a}}$ Const: Temperature kept constant at $T_{\mathrm{i}}$. Cbn: Cooling before nucleation. Han: Heating after nucleation. ${ }^{\mathrm{b}}$ Initial polymerization temperature. ${ }^{\mathrm{c}} \Delta T_{\mathrm{bn}}$ and $\Delta T_{\mathrm{an}}$ are described in Figure $1 .{ }^{\mathrm{d}}$ Numbers in parenthesis are coefficients of variation (cv). ${ }^{\mathrm{e}}$ Number of whiskers. ${ }^{f}$ not measured and calculated because the whiskers were fibrillated.

the long axis of the whisker with spiral growth caused by the screw dislocation. The whiskers are finally accomplished with the increase in the degree of polymerization by solid-state polymerization in the crystals. This formation mechanism of the whiskers is the nucleation and growth mechanism. In order to fabricate the POB nanowhiskers, two approaches are considered, which are the control of the nucleation process and the control of the growth process.

With respect to the control of the nucleation process, it is well known that critical radius of nucleus $\left(r^{*}\right)$ and nucleation rate $(J)$ depend on the degree of super saturation $(\sigma)$ as follows..$^{40,41}$

$$
\begin{aligned}
& \Delta \mu=k \mathrm{~T} \ln (1+\sigma) \quad \sigma=(C-C e) / C e \\
& r^{*}=2 v \gamma / \Delta \mu \\
& J=v_{+} q \exp \left(-16 \pi \gamma^{3} v^{2} / 3 \Delta \mu^{2} k \mathrm{~T}\right)
\end{aligned}
$$

where $\mu$ : chemical potential, $\sigma$ : degree of super saturation, $C$ : concentration of solute, $C e$ : equilibrium concentration, $r^{*}$ : critical radius of nucleus, $v$ : volume of molecule, $\gamma$ : density of surface energy, $J$ : nucleation rate, $v_{+}$: rate of crystallization of one molecule into critical nucleus, $q$ : density of free molecule. 
When the value of $\sigma$ increases, $\Delta \mu$ which is a driving force for nucleation becomes larger. This larger $\Delta \mu$ leads to smaller $r^{*}$ and larger $J$ meaning that the much more nuclei having the smaller radius are formed. The value of $\sigma$ is determined by the miscibility between oligomer and solvent, and several parameters influence the miscibility such as the solvent property, temperature and so on. The influence of the solvent property on the size of the POB whiskers was clarified that the poorer solvent made the width smaller. ${ }^{29,42} \mathrm{LPF}$ was a suitable solvent for the fabrication of the POB nanowhiskers. $\Delta \mu$ is a function of $\sigma$ which is highly correlated with the degree of supercooling in this polymerization. Self-nucleation or self-seeding is well known procedure to control the nucleation from the polymer solution, describing nucleation of a polymer melt or solution by its own crystals grown previously. ${ }^{43}$ On the basis of this consideration, the temperature drop just before the nucleation (Cbn) is likely to be workable to increase the value of $\sigma$.

With respect to the control of the growth process, previous study reveals that the step at the spiral growth is energetically favorable for the crystallization of oligomers compared with the crystallization on the side surface of the whiskers due to the advantage of the surface energy, and the oligomers are predominantly crystallized on the spiral growth step leading to the increase in the length. ${ }^{39}$ However, the oligomers start to crystallize on the side surface leading to the increase in the width when the value of $\sigma$ is quite high. It is necessary to keep the value of $\sigma$ in the suitable range for the depression of the width increase. On the basis of this discussion, the temperature increase just after the nucleation (Han) is likely to be workable to keep the value of $\sigma$.

\section{Control of Growth Process and Nucleation Process}

Firstly, the changes in the average length and width of the POB whiskers were examined in the course of polymerization at $320^{\circ} \mathrm{C}$ (Run No. 1). The results were shown in Figures 2, 3 and Table I. The length increased rapidly up to $80 \mathrm{~min}$ and then gradually to $180 \mathrm{~min}$. The length was finally $17.6 \mu \mathrm{m}$ after $6 \mathrm{~h}$. The width of the whiskers also increased slightly up to $60 \mathrm{~min}$ and afterward it became constant at $730 \mathrm{~nm}$. It is understandable that the value of $\sigma$ of the oligomer is quite high at the initial stage of polymerization and this high value of $\sigma$ results in the increase in not only the length but also the width. The polymerization temperature was raised up from 320 to $350^{\circ} \mathrm{C}$ just after the nucleation (Run No. 2) to depress the crystallization of oligomers on the side surface. The values of coefficient of variation (cv) of the length and the width of Run No. 2 were 22 and 33\%, respectively. These values were almost the same as
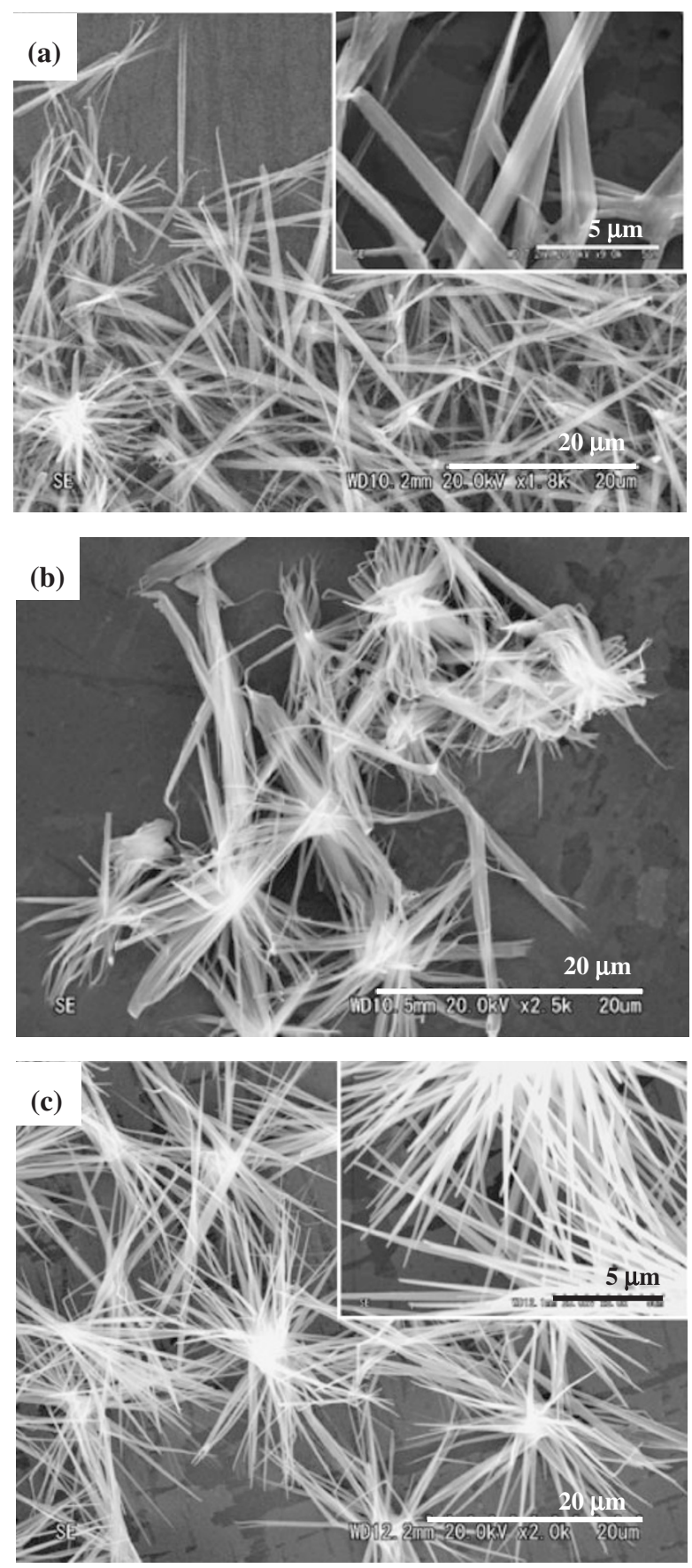

Figure 3. Morphology of $\mathrm{POB}$ whiskers prepared in (a) Run No. 1, (b) Run No. 3 and (c) Run No. 7.

those of Run No. 1. The changes in the length, width and the numbers of the whiskers $(N)$ prepared in Run No. 2 were examined in the course of the polymerization. The results were also plotted in Figure 2, and the distribution diagrams of the length and the width were presented in Figure 4. The length increased with time having unimodal distribution, whereas the width was constant at $500 \mathrm{~nm}$ maintaining unimodal distribution during polymerization. The initial increase in the width was not observed. The value of $N$ was also constant at $c a 5 \times 10^{8} \mathrm{~mL}^{-1}$ through the polymerization. The nuclei were not extinguished by the Ostwald rip- 

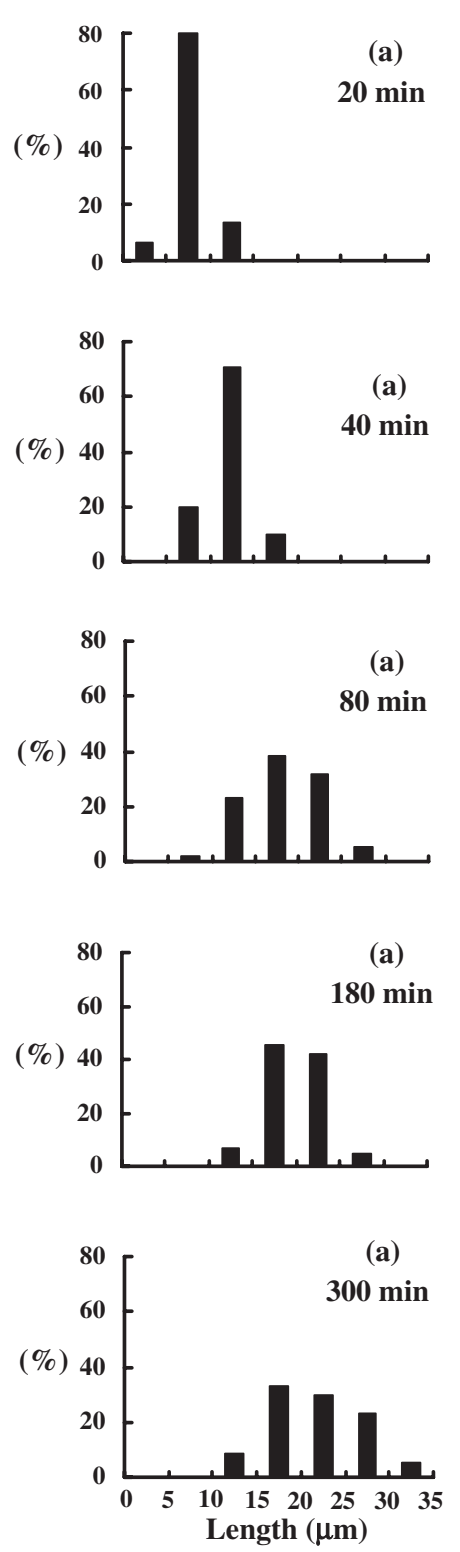
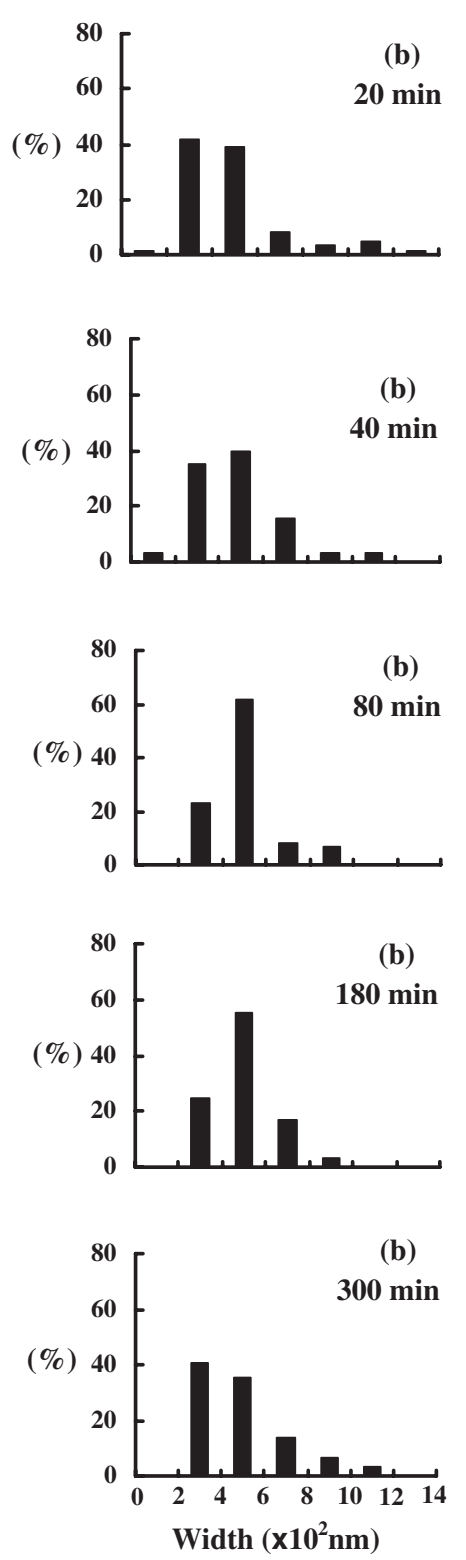

Figure 4. Distribution diagrams of (a) length and (b) width of whiskers prepared for various times in Run No. 2.

ening type dissolution and also they were not newly generated in this polymerization. The nuclei served as the whiskers were formed at the same time and the oligomers precipitated after the nucleation were consumed only for the crystal growth even in this Han process. The value of $N$ after $6 \mathrm{~h}$ in Run No. 2 was almost the same as that of Run No. 1 and the decrease in the width of Run No. 2 can be mainly attributed to the depression of the crystallization on the side surface due to the decrease in $\sigma$ by the increase in the polymerization temperature. In contrast to Run No. 2, the average width was $1.15 \mu \mathrm{m}$ when the polymerization temperature was changed from 320 to $290^{\circ} \mathrm{C}$ after the solution became turbid (Run No. 3). The whiskers obtained in Run No. 3 tended to be fibrillated as shown in Figure 3. The temperature drop after the nucleation enhanced the value of $\sigma$ leading to the increase in the width. The increase in the width during polymerization was depressed by means of the temperature increase after the nucleation.

The polymerization temperature was dropped down from 320 to $260{ }^{\circ} \mathrm{C}$ just before the solution became turbid to enhance the value of $\sigma$ leading to the decrease in the critical radius of nucleus (Run No. 4). The results are also presented in Table I. The width of the POB whiskers prepared for $6 \mathrm{~h}$ was $270 \mathrm{~nm}$. The widths of the whiskers prepared at $320^{\circ} \mathrm{C}$ (Run No. 1) and $260^{\circ} \mathrm{C}$ (Run No. 5) are 730 and $440 \mathrm{~nm}$, respectively. Although the value of $\mathrm{cv}$ of the width of Run No. 4 is slightly larger, the average width was much smaller. The value of $N$ of Run No. 4 was $50 \times 10^{8} \mathrm{~mL}^{-1}$ and this is one order magnitude over that of Run No. 1. This larger value of $N$ clearly indicates the increase in the value of $\sigma$. It is clear that 

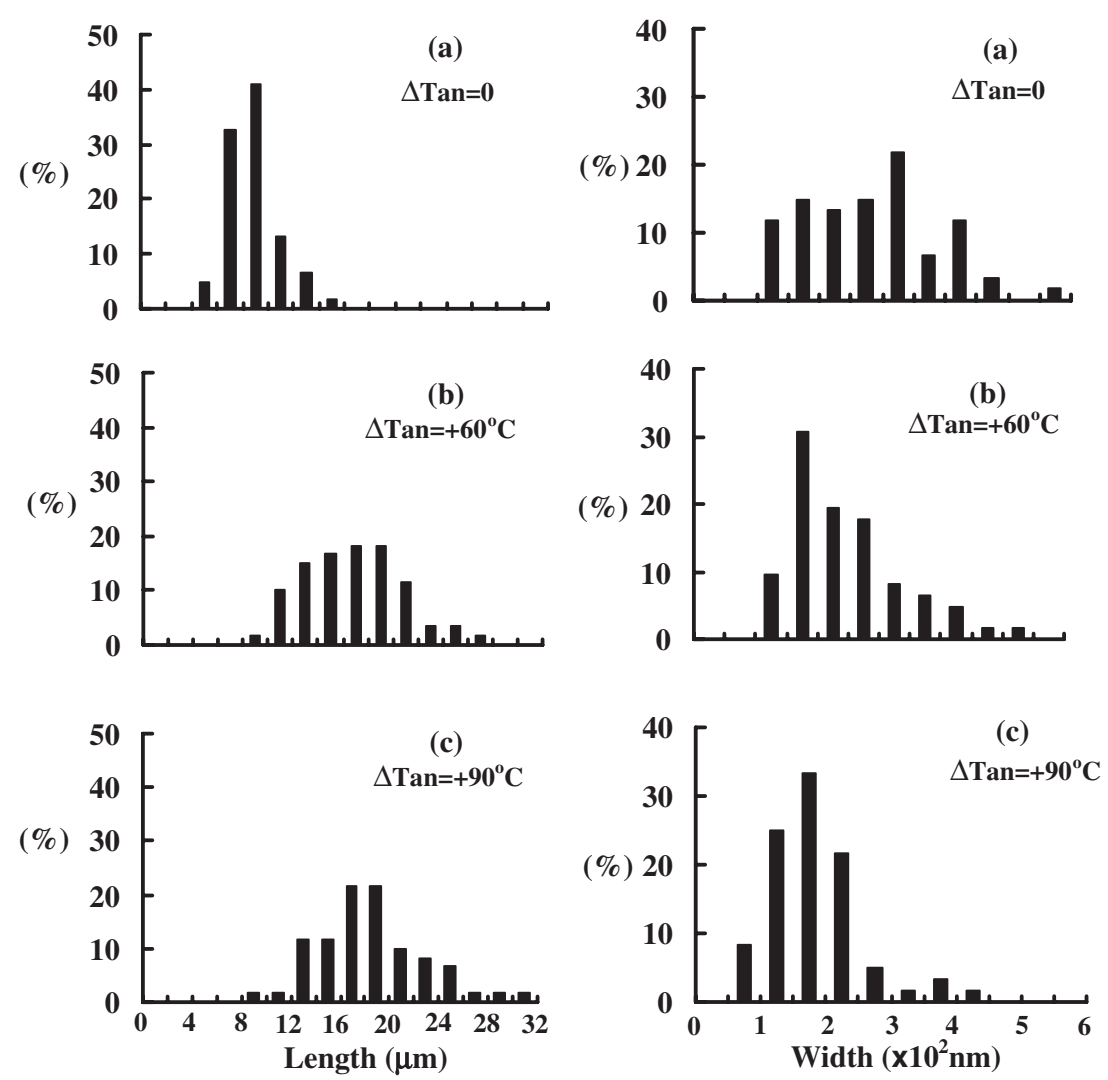

Figure 5. Distribution diagrams of length and width of whiskers prepared in (a) Run No. 4, (b) Run No. 6 and (c) Run No. 7.

the temperature drop before the nucleation is more effective to enhance the value of $\sigma$ for the nucleation compared with that of the polymerization at $260^{\circ} \mathrm{C}$.

The above discussion suggests that the most effective temperature profile to lower the width is the combination of Cbn and Han. The temperature was dropped down from 320 to $260{ }^{\circ} \mathrm{C}$ before the nucleation, heated up to $320^{\circ} \mathrm{C}$ after the nucleation and then kept at $320^{\circ} \mathrm{C}$ for $6 \mathrm{~h}$ (Run No. 6). The POB whiskers of which the width was $220 \mathrm{~nm}$ and the length was $16.0 \mu \mathrm{m}$ were obtained with the yield of $36 \%$. The value of $N$ in Run No. 6 was $46 \times 10^{8} \mathrm{~mL}^{-1}$ which was in good agreement with that of Run No. 4. In order to depress the increase in the width completely, the temperature was dropped down from 320 to $260^{\circ} \mathrm{C}$ before the nucleation and then jumped up to $350^{\circ} \mathrm{C}$ after the nucleation (Run No. 7). The width and the length of the obtained whisker were $190 \mathrm{~nm}$ and $18.6 \mu \mathrm{m}$, respectively as shown in Figure 3. In Figure 5 were shown the distribution diagrams of the length and the width of the whiskers prepared in Run No. 4, 6 and 7. They exhibited the unimodal distribution with the similar $\mathrm{cv}$ values. The length became longer and the width became smaller with the increase in $\Delta T_{\mathrm{an}}$. The value of $N$ in Run No. 7 was comparable to those in Run No. 4 and 6, which was determined by $\Delta T_{\text {bn }}$. The obtained whiskers were very symmetrical and the aspect ratio was 98 .

\section{CONCLUSIONS}

The width of the POB whiskers was very susceptible to the polymerization temperature and the change in the polymerization temperature was very efficient to control the width. Han reduced the value of $\sigma$ resulting in the depression of the increase in the width by the crystallization of oligomers on the side surface of the whiskers. Cbn enhanced the value of $\sigma$ resulting in the generation of much more nuclei having smaller width. The combination of these two methods Han and $\mathrm{Cbn}$ afforded the whiskers having the smallest width, and the POB nanowhiskers of which the width and the length were $190 \mathrm{~nm}$ and $18.6 \mu \mathrm{m}$ were prepared. The width control method by using temperature jump is surly the fundamental technology to fabricate the nanofibers of the intractable polymers.

\section{REFERENCES}

1. S. Iijima, Nature, 354, 56 (1991).

2. J. Hu, T. W. Odom, and C. M. Lieber, Acc. Chem. Res., 32, 435 (1999).

3. X. Duan, C. Niu, V. Sahi, J. Chen, J. W. Parce, S. Empedocles, and J. L. Goldman, Nature, 425, 274 (2003).

4. Z. Ying, Q. Wan, Z. T. Song, and S. L. Feng, Nanotechnology, 15, 1682 (2004). 
5. Q. Wan, C. L. Lin, X. B. Yu, and T. H. Wang, Appl. Phys. Lett., 84, 124 (2004).

6. J. F. Wang, M. S. Gudiksen, X. F. Duan, Y. Cui, and C. M. Lieber, Science, 293, 1455 (2001).

7. J. D. Holmes, K. P. Johnston, R. C. Doty, and B. A. Korgel, Science, 287, 1471 (2000).

8. Y. Wu and P. J. Tang, J. Am. Chem. Soc., 123, 3165 (2001).

9. A. L. Yarin, S. Koombhongse, and D. H. Reneker, J. Appl. Phys., 89, 3018 (2001).

10. D. H. Reneker, A. L. Yarin, H. Fong, and S. Koombhongse, J. Appl. Phys., 87, 4531 (2000).

11. M. M. Hohman, M. Shin, G. Rutledge, and M. P. Brenner, Phys. Fluids, 13, 2201 (2001).

12. M. M. Hohman, M. Shin, G. Rutledge, and M. P. Brenner, Phys. Fluids, 13, 2221 (2001).

13. R. Jaeger, M. M. Bergshoef, M. I. Battle, H. Schonherr, and G. Vancso, Macromol. Symp., 127, 141 (1998).

14. M. M. Bergshoef and G. J. Vancso, Adv. Mater., 11, 1362 (1999).

15. C. R. Martin, Chem. Mater., 8, 1739 (1996).

16. G. M. Whitesides and M. Boncheva, Proc. Natl. Acad. Sci. U.S.A., 99, 4769 (2002).

17. C. R. Lowe, Curr. Opin. Struct. Biol., 10, 428 (2000).

18. D. N. Reinhoudt and M. Crego-Calama, Science, 295, 2403 (2002).

19. J. Jang, B. Lim, J. Lee, and T. Hyeon, Chem. Commun., 83 (2001)

20. D. Xu, D. Chen, G. Guo, L. Gui, and Y. Tang, Adv. Mater., 12, 520 (2000).

21. S. H. Jeong, O. J. Lee, K. H. Lee, S. H. Oh, and C. G. Park, Chem. Mater., 14, 4003 (2002).

22. J. Lee, S. Yoon, S. M. Oh, C. H. Shin, and T. Hyeon, $A d v$. Mater., 13, 359 (2001).

23. C. R. Martin, Acc. Chem. Res., 28, 61 (1995).

24. J. Jang and J. Bae, Angew. Chem. Int. Ed., 43, 3803 (2004).

25. R. Gilkey and J. R. Caldwell, J. Appl. Polym. Sci., 2, 198 (1959).

26. K. Miyazawa, Y. Kuwasaki, A. Obayashi, and M.
Kuwabara, J. Mater. Res., 17, 83 (2002).

27. For examples;

a) P. E. Cassidy, "Thermally Stable Polymers, Syntheses and Properties," Marcel Dekker, New York, 1980.

b) A. Blumstain, "Liquid Crystalline Order in Polymers," Academic Press, New York, 1978.

28. Y. Yamashita, Y. Kato, S. Endo, and K. Kimura, Makromol. Chem. Rapid Commun., 9, 687 (1988).

29. K. Kimura, S. Endo, Y. Kato, and Y. Yamashita, Polymer, 35, 123 (1994).

30. K. Kimura and Y. Yamashita, Polymer, 35, 3311 (1994).

31. H. R. Kricheldorf and A. Conradi, Macromolecules, 22, 14 (1989).

32. G. Schwarz and H. R. Kricheldorf, Macromolecules, 24, 2829 (1991).

33. H. R. Kricheldorf, G. Schwarz, T. Adebahr, and D. J. Wilson, Macromolecules, 26, 6622 (1993).

34. H. R. Kricheldorf, G. Schwarz, and T. Adebahr, Polym. Prepr. Am. Chem. Soc. Div. Polym. Chem., 34, 768 (2003).

35. G. Schwarz and H. R. Kricheldorf, Macromolecules, 28, 3911 (1995).

36. H. R. Kricheldorf, G. Schwarz, and F. Ruhser, Macromolecules, 24, 3485 (1991).

37. H. R. Kricheldorf, O. Struve, and G. Schwarz, Polymer, 37, 4311 (1996).

38. A. K. Sarker, K. Kimura, F. Yokoyama, and Y. Yamashita, High Perform. Polym., 13, 351 (2001).

39. Y. Yamashita and K. Kimura, "Polymeric Materials Encyclopedia,” CRC Press: Boca Raton, 1996, p 8707.

40. W. K. Burton, N. Cabrera, and F. C. Frank, Philos. Trans. R. Soc. London, Ser. A, 243, 299 (1950).

41. F. C. Frank, Adv. Phys., 1, 91 (1952).

42. K. Kimura, A. K. Sarker, H. Ohmori, and Y. Yamashita, J. Appl. Polym. Sci., 87, 1129 (2003).

43. B. Wunderlich, "Macromolecular Physics, Vol. 2. Crystal Nucleation, Growth, Annealing," Academic Press, New York, 1976. 